\title{
Experts set top priorities for oral health research
}

Dental health professionals, members of the public, patients, and carers have for the first time worked together to identify the most pressing unanswered research questions on how to improve oral and dental health for individual patients, communities and the population.

In 2017, a partnership between the National Institute for Health Research (NIHR) Clinical Research Network Oral and Dental Health Specialty Group in collaboration with the Dental Schools Council and Public Health England (PHE) was launched to identify unanswered questions relating to oral and dental health research from a patient, public and clinical perspective.

The work has taken 18 months to complete and identified gaps in knowledge about oral and dental care using a method tested by the James Lind Alliance, the non-profit making initiative established to bring patients, carers and clinicians together in Priority Setting Partnerships (PSPs) to identify and prioritise unanswered questions or evidence uncertainties.

Despite improvements, dental health remains a major public health problem, according to the NIHR, pointing to the fact that a quarter of five-year-olds in England have had tooth decay and nearly three times as many children from the poorest families are affected than those from the richest.

The new priorities, ${ }^{1}$ published on 19 December 2018, are:

- What is the best way to prevent tooth decay, and reduce oral health inequalities at a community or population level?

- How can access to dental services be improved for the general public?

- What are the most effective ways of increasing early detection/ diagnosis of oral cancer?
- How can access to dental services be improved for people with additional needs?

- How can dental health professionals work with other health professionals to help improve oral health?

- How can basic oral hygiene be achieved for people with additional care needs?

- How to improve communication between dental teams and patients/carers?

- Is there a role for dental health professionals in treating oral health problems to improve general health?

- What is the best way to prevent gum disease, and reduce oral health inequalities at a community or population level?

- What role do digital technologies play in the provision of dental care?

Professor Peter Robinson of the University of Bristol and the NIHR who led the Priority Setting Partnership said: 'It is hugely important to have worked with our patients and the general public to have identified these Top 10 questions.

'With 60 million patients nationally and so many different diseases to consider, these priorities will help researchers concentrate on the most pressing problems. Agencies who fund research have already said they will focus their efforts on the topics we have identified today, that means the questions we have identified today will now be answered in the next few years.'

1. National Institute for Health Research. The Top 10 Priorities for Oral and Dental Health Research. 2018. Available at https://www.nihr.ac.uk/nihr-in-your-area/oral/documents/ JLAOralandDentalPSPFinalTop10Priorities.pdf (accessed January 2019).

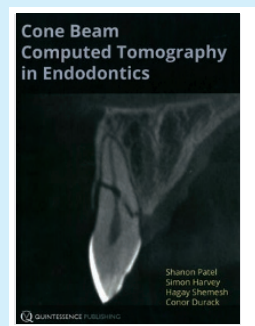

\section{CONE BEAM COMPUTED TOMOGRAPHY IN ENDODONTICS}

Shanon Patel, Simon Harvey, Hagay Shemesh, Conor Durack

2016; Quintessence; price $\mathrm{f} 65.00$ pp. 144

ISBN: 9781850972914

This is a comprehensive book focusing on the use of cone beam computed tomography (CBCT) within endodontics, providing evidence, guidance and rationale. The contributors comprise of a wide range of international experts within the field of endodontics and dental and maxillofacial radiology. This makes it an ideal resource for specialists in endodontology and dental and maxillofacial radiology, general dentists and undergraduates.

The book is divided into two sections. The first section consists of four chapters which provide an overview on the limitations of conventional radiography, radiation physics, and CBCT and its uses. This section of the book is ideal for those looking for a revision tool or to find out more on the essential aspects of radiology relating to CBCT. At the end of these chapters the reader is also provided with guidance on further reading that can be performed. The second section of the book consists of seven chapters and moves on to cover the specific applications of $\mathrm{CBCT}$ in endodontics, including dentoalveolar and root canal anatomy, endodontic diseases and endodontic therapies. These chapters all end with a referencing section providing the reader with evidences to the information covered, and act as a guide to further resources.

All of the chapters contain high quality images, illustrations and diagrams which are relevant and enable the reader to gain a deeper and more focused understanding of $\mathrm{CBCT}$ in endodontics. Each chapter also provides the reader with sufficient detail, offering a greater sense of guidance and comprehension of $\mathrm{CBCT}$ in endodontics.

I believe that this book is an invaluable resource for all levels of clinicians especially those within speciality training posts of endodontology and dental and maxillofacial radiology. It also serves as a good resource for undergraduates. I would strongly advise those seeking a deeper understanding of $\mathrm{CBCT}$ in endodontics to read this book as it will no doubt greatly increase their understanding and appreciation of this area of dentistry.

Jasem Greval 